\title{
In varietate concordia oder Abrechnungsmentalität unter Kollegen
}

\section{Bernhard Schaller ${ }^{a}$, Gaby Baller ${ }^{b}$}

a Universitätsklinikum Münster/D

b GMHM mbH, Berlin, Mannheim, Heidelberg; Institut für Medizincontrolling, Heidelberg/D; www.drg24.ch
1 Watzlawick P. Anleitung zum Unglücklichsein. München: Piper; 1983.

2 Watzlawick P. Menschliche Kommunikation. Formen, Störungen, Paradoxien. Bern 1969.

3 Diese Konvention geht auf Gerold Ungeheuer zurück.
Das Gesundheitswesen ist im Wandel wie wohl nie zuvor in der Geschichte der Menschheit, und es besteht ein zunehmender Trend zu stärkerer Ökonomisierung. Damit ist auch eine Intensivierung des Wettbewerbs zwischen den Krankenhäusern einerseits und zwischen den Ärzten andererseits verbunden. Es fällt daher auf, dass die Schärfe der Auseinandersetzung zwischen den verschiedenen Interessengruppen zugenommen hat. Dies hat uns dazu bewogen, einige Überlegungen zur Kommunikation anzustellen. Wir sehen dabei den Begriff Kommunikation im Zusammenhang mit dem der Interaktion. Vor diesem Hintergrund versuchen wir, die Parallelen zwischen Kommunikation und Verhalten herauszuarbeiten.

Paul Watzlawick war ein Kommunikationswissenschaftler, der durch seine wissenschaftlichen Veröffentlichungen zur Kommunikationstheorie und über den radikalen Konstruktivismus bekannt wurde. Watzlawick hat Heraklits Gedanken von der «Einheit in der Vielfalt» der Dinge - Enantiodromie - aufgegriffen und darauf hingewiesen, dass ein Zuviel des Guten stets ins Böse umschlage. Zuviel Patriotismus erzeuge Chauvinismus, zuviel Sicherheit Zwang oder zuviel Buttercremetorte Übelkeit. Nach Clifford A. Pickover ist Enantiodromie darüber hinaus der Prozess, in dem sich ein Glaube in sein Gegenteil verwandelt. Pickover nennt als Beispiel Paulus von Tarsus.

Besonders bekannt wurde folgendes Beispiel aus der «Anleitung zum Unglücklichsein» [1]. Darin beschreibt Watzlawick einen Mann, der alle zehn Sekunden in die Hände klatscht. Nach dem Grund für dieses merkwürdige Verhalten befragt, erklärt er: «Um die Elefanten zu verscheuchen.» Auf den Hinweis, es gebe hier doch gar keine Elefanten, antwortet der Mann: «Na, also! Sehen Sie?» Damit wollte Watzlawick zeigen, dass der konsequente Versuch, ein Problem zu vermeiden - hier die Konfrontation mit Elefanten -, es in Wirklichkeit verewigt.

Das zugegebenermassen eindrucksvolle Beispiel von Watzlawick zeigt die Bedeutung der Kommunikation heutzutage in ihrer ursprünglichen Bedeutung als wechselseitiges Übermitteln von Daten oder von Signalen, die für den Beobachter der Kommunikation eine festgelegte Be- deutung haben. Die Signale gelten dann als Auslöser für bestimmte Reaktionen. Dies betrifft auch tierische und pflanzliche Lebewesen, wenn diese naturwissenschaftlich beobachtet werden (zum Beispiel als molekulare Körper), sowie technische Objekte oder Systeme.

Das Wort Kommunikation fand erst Anfang der 1970er Jahre Eingang in den deutschen soziologischen Sprachgebrauch. Die Psychologen Paul Watzlawick, Don D. Jackson und Janet H. Beavin behandelten 1967 aus therapeutischer Sicht die Rolle von Kommunikation in zwischenmenschlichen Beziehungen. Im Vorwort zur deutschen Ausgabe seines Werks «Menschliche Kommunikation. Formen, Störungen, Paradoxien» bezeichnet Watzlawick den Begriff Kommunikation als «im Deutschen ungewohnt» [2].

Wenn davon ausgegangen wird, dass Kommunikation der gemeinschaftlichen Problemlösung dient, können die angestrebten Problemlösungen unter zwei Aspekten beschrieben werden. Im einen Fall werden diejenigen Problemlösungen betrachtet, die zwischen den Kommunizierenden eintreten sollen - beispielsweise eine Änderung von Überzeugungen, Wissen, Meinungen und Vorstellungen. Im anderen Fall werden diejenigen Problemlösungen betrachtet, die in der gemeinsamen Umgebung eintreten sollen. Beide Fälle gehören zu den Problemlösungen, die nicht von einem einzelnen allein erreicht werden können, zu denen also Kommunikation notwendig ist. Eine Konvention besteht darin, im ersten Fall die angestrebte Lösung (die Verständigung) als Kommunikationsziel, im zweiten Fall die angestrebte Lösung (die Wirkung in der Umgebung) als Kommunikationszweck zu bezeichnen. Das Kommunikationsziel ist dann primär, der Kommunikationszweck sekundär oder übergeordnet [3].

Die gewaltfreie Kommunikation ist eine von Marshall B. Rosenberg entwickelte Kommunikations- und Konfliktlösungsmethode, die zur Absicht hat, in einen Kontakt mit anderen beziehungsweise mit sich selbst zu kommen, in dem Geben und Nehmen ein fliessender Prozess ist und freiwillig geschieht. Es geht also nicht (nur) darum, andere dazu zu bringen, zu tun, was man selber will. Ziel ist es, die Anliegen aller Parteien aufzuspüren und zu berücksichtigen. Rosenberg 
geht davon aus, dass die Form, in der wir miteinander kommunizieren, einen entscheidenden Einfluss darauf hat, ob wir Empathie für unser Gegenüber entwickeln und unsere Bedürfnisse erfüllen können. Er nennt dies «mit dem Leben tanzen». Im Zentrum steht ein Miteinander, das nicht verurteilt, sondern auf die Bedürfnisse und Gefühle gerichtet ist, die hinter Handlungen und Konflikten stehen. Das Konzept der gewaltfreien Kommunikation kann in vielen Bereichen verwendet werden, so etwa ich im alltäglichen Umgang unter Ärztekollegen. Die gewaltfreie Kommunikation ist jedoch weniger als Kommunikationstechnik zu betrachten, sondern als Bewusstwerdung über Möglichkeiten des empathischen Kontaktes. So reicht es nach Rosenberg dann nicht, das Grundmodell stur anzuwenden, sondern es ist eine veränderte Einstellung zum Gegenüber notwendig, um die Kommunikation und das Wohlbefinden zu verbessern.

Schon die Mäeutik des Sokrates will nicht die Wertung vollkommen bannen, sondern stellt ihren Nutzen in würdigender Form zur Verfügung. Aus systemischer Sicht gehören Provokation, Machtdemonstration und Wettbewerb zum menschlichen Erleben. Aus Sicht der themenzentrierten Interaktion wird empfohlen, die eigenen Interpretationen so lange wie möglich zurückzuhalten, jedoch wird als Axiom gesetzt, dass Bewertung notwendig ist. Auch im Bereich der Theorien von Deeskalation und Konfliktmanagement finden sich Konzepte, die bewertende Interventionen mit Erfolg einsetzen. Nicht zuletzt fordert das aus dem Businessbereich stammende sogenannte Harvard-Konzept explizit sogar «Hart in der Sache - weich zum Menschen».

Die gewaltfreie Kommunikation steht in der Tradition der klientenzentrierten Gesprächstherapie, die von Rosenbergs Lehrer Carl Rogers entwickelt wurde. Das aktive Zuhören steht bei Rogers im Mittelpunkt, die gewaltfreie Kommunikation geht jedoch über den gesprächstherapeutischen Rahmen hinaus. Beeinflusst ist die gewaltfreie Kommunikation auch durch Ghandi und seine Überlegungen zur Gewaltfreiheit, Ahimsa genannt, die auf den Upanischaden basieren. Viele Elemente der gewaltfreien Kommunikation finden sich auch in anderen Konfliktlösungstechniken, wie in der Mediation und den Win-win-Strategien. Die Gewaltfreiheit geht davon aus, dass Gewalt oder deren Androhung Probleme nicht lösen, Ungerechtigkeit und Unterdrückung nicht beseitigen kann. Daher wurden vielfältige Aktionsmethoden entwickelt, um gewaltfreie Konfliktlösungen zu erreichen.

Mahatma Ghandi schrieb: «Die Grundbedeutung von Gewaltfreiheit ist Festhalten an der
Wahrheit, Kraft der Wahrheit (Satyagraha). [...] Bei der Anwendung von Gewaltfreiheit entdeckte ich schon sehr früh, dass die Wahrheitssuche es nicht erlaubt, dem Gegner Gewalt anzutun. Er muss vielmehr durch Geduld und Mitgefühl von seinem Irrtum abgebracht werden.»

Politische Korrektheit ist ein aus dem angelsächsischen Raum stammendes Schlagwort, das wohl bekannter als der Begriff gewaltfreie Kommunikation ist, aber auch zeigt, dass gerade diese Sprachregion uns diesbezüglich voraus ist. Die politische Korrektheit stand ursprünglich im positiven Sinne für Antidiskriminierungsbemühungen in den USA. Heute dient die Bezeichnung vorrangig als Begriff gegen verschiedene sprachliche Konventionen und Verhaltensweisen, die nach Ansicht ihrer Befürworter den Abbau von Diskriminierung nach dem Geschlecht oder gegenüber sozialen, religiösen oder nationalen Minderheiten zum Ziel haben, nach Ansicht ihrer Gegner aber oft zumindest übertrieben oder unpraktikabel sind. Im deutschsprachigen Raum wurde der Neologismus von konservativer Seite importiert [4] und soll nach Elisabeth Noelle-Neumann als Indiz für einen «Konformitätsdruck» bei bestimmten «Themen gelten, mit denen die Gesellschaft noch nicht fertig geworden ist» und die deshalb als «heikel» empfunden werden und so eine «Tabuisierung» begründen, die eine offene Diskussion verhindere [5].

In seinem 1963 erschienenen Bestseller «Das sogenannte Böse. Zur Naturgeschichte der Aggression» schreibt der Arzt und Verhaltensforscher Konrad Lorenz dass «ein Tier in einer von Angst gekennzeichneten Situation flieht oder angreift». Sein Schüler Irenäus Eibl-Eibesfeldt, der einer der meistgeachteten Forscher auf dem Gebiet der Verhaltensbiologie ist, übertrug diese Theorie später auf das menschliche Verhalten. Deshalb scheint uns aggressives Verhalten oder Kommunizieren aus einer Angst heraus resultierend. Welche Ängste hier zugrunde liegen, wagen wir nur zu ahnen: Angst vor Veränderungen, Angst vor finanziellen Einbussen, Angst vor Überforderung, droht ein Verlust des Arbeitsplatzes, oder befürchtet man den Verlust von persönlichen Entscheidungsbefugnissen oder Handlungsspielräumen?

Wenn offen über die Hauptursachen der Ängste gesprochen wird, ist der Weg frei für das Aushandeln von Vorgehensweisen, die den Interessen der Betroffenen Rechnung tragen, möglicherweise sind deren Befürchtungen nicht gerechtfertigt.

Man mag sich nun fragen, was dies nun mit dem Verhalten der Ärzte untereinander zu tun hat. Die Schweizer Ärzteschaft ist eine Organisa- 
tion, die in deutlicher Veränderung begriffen ist. Was gestern noch als richtig gegolten hat, wird heute bereits in Frage gestellt und morgen vielleicht schon geändert. Die Managementlehre kennt eine sogenannte «Delphinstrategie», die in den USA von Dudley Lynch und Paul Kordis entwickelt wurde. Die Erfinder der Delphinstrategie gehen davon aus, dass Delphine ihre Verhaltensweisen schnell und präzise im Team ändern, wenn sie nicht erreichen, was sie anstreben. Diese Fähigkeiten haben Dudley Lynch und Paul Kordis beobachtet und sinnbildlich auf Menschen und Organisationen im Umgang mit Veränderung übertragen. Eine wesentliche Aussage ist, dass Veränderungen in konventionell geführten Organisationen - wie es ein Krankenhaus ist - gewöhnlich zu spät vorgenommen werden. Das habe zur Folge, dass bei konventionellem Management vermeidbare Ertrags- und Energieverluste auftreten, die sich durchaus in einer Brutalität des Umgangs zwischen den verschiedenen Berufsgruppen einerseits, aber auch innerhalb der einzelnen Berufsgruppen andererseits manifestieren. Dass dabei die Sprache als wichtigste Kommunikationsform des Menschen ein entscheidendes Glied in der Kette darstellt, liegt - so glauben wir - auf der Hand. Das Symbolbild des Delphins weist hierbei auf eine Gemeinschaft hin, in der sowohl jedes einzelne Mitglied seine Individualität behält als auch gleichzeitig seine Fähigkeiten als Teil eines Ganzen optimal einsetzt.

Im Jahre 1998 hat die Vollversammlung der Vereinten Nationen das erste Jahrzehnt des 21. Jahrhunderts und des 3. Jahrtausends, die Jahre 2001-2010, zur Internationalen Dekade für eine Kultur des Friedens und der Gewaltfreiheit für die Kinder der Welt deklariert. In diesem Sinne ist es zu wünschen, dass auch wir Ärzte gerade in der Diskussion untereinander, wo die Partikularinteressen unterschiedlicher nicht sein könnten, lernen, eine gewaltfreie Kommunikation anzuwenden: Unser Berufsstand hat eine eigene Standesmoral entwickelt, deren bekannteste Form der Eid des Hippokrates ist, der heute in der Genfer Deklaration des Weltärztebundes weiterlebt. Was darin für den Umgang mit Patienten steht, kann durchaus auf für den modernen zwischenmenschlichen Umgang unter Kollegen gelten, der da besagt, dass man sich «nicht beeinflussen lassen» soll «durch Alter, Krankheit oder Behinderung, Konfession, ethnische Herkunft, Geschlecht, Staatsangehörigkeit, politische Zugehörigkeit, Rasse, sexuelle Orientierung oder soziale Stellung». Derzeit bewegen wir Ärzte uns gezielt in einer Atmosphäre der kultiviert verdeckten Feindseligkeit und mit grosser Freude am Gewinner-Verlierer-Prinzip. So ist auch die entsprechende Kommunikation häufig deutlich von Konzepten geprägt, in dem z. B. die persönlichen Elemente einer Sachdiskussion in den Mittelpunkt der Auseinandersetzung rücken, um Einfluss und Verantwortung auf Kosten persönlicher Defizite eines Konkurrenten zu vergrössern. Eines der grundlegendsten Konzepte der Win-win-Problemlösungen wurde in den 1970 und 1980er Jahren an der Harvard Universität im Rahmen des dort angesiedelten «Harvard Negotiation Project» entwickelt, in dem die Entwicklung verbesserter Verhandlungsmethoden wissenschaftlich untersucht wurde. Die Methode des «sachgerechten Verhandelns» wurde als «Harvard-Konzept» bekannt und liegt vielen Ansätzen zur Konfliktlösung wie z. B. der Mediation zugrunde. Es geht dabei nicht darum, die eigene Position durchzusetzen oder gezwungenermassen Abstriche zu machen, sondern darum, eine dauerhafte Lösung zu finden, die von allen Beteiligten getragen und akzeptiert wird. Hier wird eine Situation geschaffen, in der jeder die Wahrnehmung und auch das Gefühl hat, durch diese Lösung etwas zu gewinnen und nicht zu verlieren.

Zusammenfassend, und dies scheint mir auch im Hinblick auf den Umgang unter Kollegen wichtig, können obige Ausführungen in das Konzept der emotionalen Kompetenz integriert werden, das ursprünglich von Salovey und Mayer stammt [6]. Als emotionale Kompetenz wird die Fähigkeit verstanden, mit eigenen und fremden Gefühlen umzugehen, diese im alltäglichen Kontext richtig zu bewerten und so Konflikte und Stress zu vermeiden. Ziel des Handelns ist dann die Positivität des Fühlens, wie sie sich in allen Arten von Werterfahrungen äussern kann, und zwar innerhalb des emotionalen Systems am effektivsten, wenn dabei für alle Seiten dauerhafte, zuverlässige und für die Beteiligten nützliche, angenehme, freudvolle und produktive Beziehungen erreicht werden. Dies ist einer der Faktoren, die nach heutigem Verständnis über den beruflichen Erfolg entscheiden und wohl auch im Fall der Schweizer Ärzte als Gesamtheit entscheiden werden, wie sie sich als Berufsgruppe im veränderten Gesundheitsumfeld plazieren können. Nicht zuletzt wäre dies doch ganz im Sinne eines respektvollen Umgangs unter (ärztlichen) Kollegen, wie er eigentlich selbstverständlich sein müsste und auch nicht zuletzt im Eid des Hippokrates gefordert wird. Denn nur gemeinsam können wir etwas bewegen!
Intelligence. Imagination Cognition, and Personality. 1990;9:185-211. 